\title{
Realizing the Dream: The Future of Primary Care Research
}

Arlene S. Bierman, MD, MS

Sebastian T. Tong, MD, MPH

Robert J. McNellis, MPH, PA

Center for Evidence and Practice Improvement, Agency for Healthcare Research and Quality, Rockville, Maryland

Conflicts of interest: authors report none.

\section{CORRESPONDING AUTHOR}

Arlene S. Bierman

Center for Evidence and Practice Improvement, Agency for Healthcare Research and Quality 5600 Fishers Lane

Rockville, Maryland 20857

arlene.bierman@ahrq.hhs.gov

\begin{abstract}
Primary care research is central to the successful transformation of care delivery, providing the crucial evidence to overcome the longstanding and widespread threats and challenges to the realization of primary care's full potential. The Agency for Healthcare Research and Quality (AHRQ), as the federal agency specifically charged with conducting and supporting primary care research, plays a pivotal role in supporting the research and generating the evidence needed to advance primary care. Drawing upon decades of AHRQ-supported research studies, extensive stakeholder consultation, and a Primary Care Research Summit held in fall 2020, we discuss the primary care research central to successful primary care transformation and for realizing the vision of a high-performing US health system to effectively serve all Americans and their communities while advancing health equity.

Realizing the potential of primary care will require wise investments in primary care research. Newly generated evidence needs to be rapidly incorporated into the design of the delivery system, clinical care, and community interventions. Investments in evidenceinformed primary care redesign can catalyze progress to achieving the quintuple aimimproved health outcomes, increased value, better patient and clinician experience, and health equity. Primary care research can provide the evidence to help stem the twin epidemics of clinician burnout and lack of trust in the health system. Actualizing this vision will require a concerted and coordinated effort by policy makers, researchers, clinicians, and community members and a commitment to ensuring people and communities have ready access to primary care.
\end{abstract}

Ann Fam Med 2022;20:Online. https://doi.org/10.1370/afm.2788.

Annals "Online First" article. Accepted for publication in a later issue.

\section{A VISION OF THE FUTURE}

$\mathrm{t}$ is 2032. After a near-death experience, primary care is thriving and has achieved its rightful place at the center of the US health system. Transdisci-

plinary teams, focused on wellness and whole person health, have developed ongoing, trusting relationships with the people and communities they serve and are seamlessly integrated with the rest of the health system, public health, and human services. Primary care routinely collaborates with other sectors integral to the production of health, including education, housing, and transportation. With burnout decreasing and joy in practice thriving, health professional students are entering primary care at record levels.

This remarkable transformation has produced considerable return on investment for policy makers, payers, and health system leaders-including for themselves and their families - as the burden of navigating the health system and coordinating care has been greatly reduced. All people can readily access primary care, and, as a result, health inequities have been significantly reduced and life expectancy declines have reversed. ${ }^{1}$

Lessons learned from the COVID-19 pandemic together with several influential reports spurred a decade of investment, innovation, and research in primary care. ${ }^{2-4}$ Findings from the 2019 RAND report prompted purposeful coordination of primary care research across federal agencies and drew attention to the many evidence gaps that primary care research was needed to fill. ${ }^{2}$ The National Academy of Sciences, Engineering, and Medicine report, Implementing High-Quality Primary Care, catalyzed long-needed reforms to advance primary care. ${ }^{3}$ The World Bank report declared that it was time to deliver globally on the long-recognized promise of primary health care. ${ }^{4}$ A new generation of primary care researchers built upon decades of prior research to generate the critical evidence on how to consistently deliver accessible, high quality, effective, and equitable care. Widespread adoption of the 
Care and Learn model ${ }^{5}$ supported the evolution of primary care practices and networks to become learning health systems. Learning collaboratives supported the spread of innovation and evidence.

This commitment to innovative primary care research made all the difference. Coproduction of evidence by people seeking care, their families and caregivers, clinicians, and communities became an integral part of research. Innovative study design and research methods, availability and accessibility of needed data, and the integration of quality improvement and implementation science supported the generation of practice-based evidence. Together, these activities greatly enhanced the value of research investments, creating a world where evidence synthesis, generation, and implementation were integrally embedded into the work of care delivery.

\section{Research Needed to Realize the Potential of Primary Care}

Primary care research, which is central to the transformation of care delivery, can help realize this vision by providing the evidence to overcome long-standing and widespread threats and challenges to the realization of primary care's full potential. The Agency for Healthcare Research and Quality (AHRQ), as the federal agency specifically charged with conducting and supporting primary care research, plays a pivotal role in supporting the research and generating the evidence needed to advance primary care. Drawing on decades of AHRQ-supported studies, extensive stakeholder consultation, and a Primary Care Research Summit held in fall 2020, we discuss the primary care research central to successful primary care transformation and for realizing the vision of a high-performing US health system that advances health equity to effectively serve all Americans and their communities. AHRQ has identified core attributes of primary care with key themes (Table 1) where research is needed to achieve this vision for the future of primary care.

\section{Core Attributes of Primary Care}

Person and Family Centeredness

To implement person- and family-centered care, ${ }^{3,6}$ primary care needs to build continuous, trusting relationships in which clinicians, patients, and families are partners in care planning. The organization, financing, and delivery of care to promote wellness, prevent disease, and manage illness needs to support models of care that enable primary care practices to deliver comprehensive and continuous whole person care rather than primarily focus on specific diseases and services, and engage patients in coproduction of health and in shared decision making. It is essential that patients are involved in research as partners and play an integral role in identifying research priorities, meaningful outcomes to measure, and ways to redesign care. Meaningfully engaging patients will foster the implementation of person centeredness in primary care.

\section{Community Centeredness}

Primary care practices should play an integral role in improving and promoting the health of the communities they serve. Community centeredness can be fostered by creating linkages

\section{Table 1. Core Attributes of Primary Care, Definitions, and Examples}

\begin{tabular}{|c|c|c|}
\hline Attribute & Definition & $\begin{array}{l}\text { Examples of AHRQ Resources } \\
\text { to Guide Research }\end{array}$ \\
\hline $\begin{array}{l}\text { Person and family } \\
\text { centeredness }\end{array}$ & $\begin{array}{l}\text { Person and family centeredness entails caring for people in the context of their } \\
\text { lived family, social, and community experiences. It refers to being "respectful } \\
\text { of and responsive to individual patient preferences, needs and values." }{ }^{3,6}\end{array}$ & $\begin{array}{l}\text { Chartbook on Person- and Family- } \\
\text { Centered Care }\end{array}$ \\
\hline Community centeredness & $\begin{array}{l}\text { Primary care practices should play an integral role in improving and promot- } \\
\text { ing the health of the communities they serve by engaging with community } \\
\text { organizations with a shared mission. }\end{array}$ & $\begin{array}{l}\text { Clinical-Community Relationships } \\
\text { Measures Atlas }\end{array}$ \\
\hline Primary care centeredness & $\begin{array}{l}\text { Primary care is the foundation for all other health care. All individuals would } \\
\text { have access to and receive comprehensive, longitudinal, and coordinated, } \\
\text { high-quality primary care. }\end{array}$ & $\begin{array}{l}\text { Redefining Primary Care for the } \\
21^{\text {st }} \text { Century }\end{array}$ \\
\hline Advancing health equity & $\begin{array}{l}\text { Primary care has a critical role in eliminating pervasive, structural, and long- } \\
\text { standing inequities in access, quality, and outcomes of care. }\end{array}$ & $\begin{array}{l}\text { A Call to Action to Achieve Health } \\
\text { Equity }\end{array}$ \\
\hline Digital health solutions & $\begin{array}{l}\text { Ongoing advancements in health information technology and the development } \\
\text { of digital health solutions designed specifically to support primary care. }\end{array}$ & $\begin{array}{l}\text { AHRQ's Digital Solutions to Sup- } \\
\text { port Care Transitions Challenge }{ }^{11}\end{array}$ \\
\hline Aligning payment & $\begin{array}{l}\text { Fundamental transformation of primary care is not possible without equally } \\
\text { fundamental changes in the allocation of resources to primary care. }\end{array}$ & $\begin{array}{l}\text { New Models of Primary Care } \\
\text { Workforce and Financing }{ }^{12}\end{array}$ \\
\hline Workforce development & $\begin{array}{l}\text { Primary care teams are designed to allow all team members to practice at the } \\
\text { "top of their license," and can continuously work to improve patient care, } \\
\text { while reducing individual workloads and burnout. }\end{array}$ & $\begin{array}{l}\text { Creating Patient-Centered Team- } \\
\text { Based Primary Care }{ }^{13}\end{array}$ \\
\hline
\end{tabular}


with community-based organizations and services, public health, and other sectors that influence health, such as education, housing, and transportation. Community members as practice partners can inform the design and operation of the practice, bringing their intimate knowledge of community needs and local resources. ${ }^{14}$ Innovative research to design, implement, and evaluate clinical-community-partnered interventions that align the clinical management role of primary care practices with evidence-based community approaches to address population health can advance this aim. Investment in outreach and relationship building will be required. Community-oriented primary care can be further enabled by practices' access to available data on the health of the community served and available community resources. To make the best use of these data, practices will require easy-to-use tools. Research on how to best create bidirectional information flow between practices and public health will be as essential to addressing the next pandemic or natural disaster as to addressing chronic disease prevention and management.

\section{Primary Care Centeredness}

In a primary care-centered health system, all individuals would receive comprehensive, longitudinal, and coordinated care anchored in primary care. Ideally, primary care clinicians with a holistic perspective of the people for whom they provide care will coordinate and integrate that care with specialty care, behavioral health, home care, acute care, and post-acute care. Primary care practices would serve as the medical home, integrated with the broader "medical neighborhood," where patients seek their care, trust their clinicians, and value continuity. Together, patients and primary care clinicians can determine when expertise of specialist clinicians is needed. Such bidirectional communication with specialty care would include shared and stepped-care approaches to management, with patient goals and preferences informing decision making. Transitions across providers and settings of care would be designed to optimize outcomes and avoid errors of omission and/or duplication due to poor information sharing and communications. Research is needed on the models of care necessary to actualize primary care-centeredness. Such models would ensure that patients receive the best care that aligns with their goals, values, and expectations.

\section{Advancing Health Equity}

Primary care should seek to eliminate pervasive and long-standing inequities in access, quality, and outcomes of care. Increasing access to effective health care for racial/ethnic minorities, low-income and rural communities, and other populations experiencing disparities is necessary, as are targeted improvement interventions. Quality improvement interventions can narrow, maintain, or widen health inequities depending on their relative effectiveness in different populations. Populations and communities that currently experience suboptimal health outcomes, unequal treatment, bias, or discrimination must be active partners in codesigning effective interventions and coproducing the evidence needed to eliminate health inequities. Success will require researchers to not only intentionally recruit participants in primary care studies who represent populations served, but also engage members of the community as stakeholders and leaders in those studies. This participatory and partnership research along with the mainstreaming of health equity goals in study design can assure that society's investments in primary care transformation and research benefit all. ${ }^{15}$

\section{Digital Health Solutions}

Ongoing advancements in health information technology and the development of digital health solutions tailored to primary care practice can enable the desired renaissance in primary care delivery. User-centered digital health applications designed to facilitate patient care, self-management support, care transitions and coordination, and population health, all have the potential to improve care delivery while reducing the burden on clinicians, primary care teams, patients, and caregivers. User-centered design, agile implementation, and rigorous testing and evaluation can be used to develop interoperable, user-friendly electronic health records designed to support patient care, quality improvement, and population health management.

Advances in clinician and patient-facing clinical decision support (CDS), together with implementation research on how to effectively integrate CDS tools into workflows, will increase access to the best evidence at the point of care to inform decision making and improve quality. Research can also provide advances in our understanding of how to structure patient-clinician encounters that bring together patient-generated health data, remote monitoring, synchronous and asynchronous virtual care, and data from other non-visit-based encounters. Interoperable shared e-care plans can facilitate management of individuals with complex physical, mental health, and social needs, especially those who are older, frail, and/or have multiple chronic conditions. Research is also needed on the role of digital health solutions within multicomponent improvement interventions.

\section{Aligning Payment}

Fundamental transformation of primary care is not possible without equally fundamental changes in the allocation of resources to primary care. Supporting the core attributes of primary care will require payment policies that align incentives with desired outcomes. Evidence is needed for payment models that support team-based care and allow practices to engage fully in community-centered care, advancing health equity, and incorporating digital health solutions. Studying diverse states' experiences with implementing payment reform to support primary care can lead to the adoption of effective approaches to increase the proportion of health care spending allocated to primary care. ${ }^{16}$ Researchers in partnership with primary care practices and the communities they serve need to learn what types of technical assistance are necessary to finally transition away from the fee-for-service paradigm to payment 
mechanisms that support whole-person health. We need measures to assess the effectiveness of these alternative payment models in supporting the delivery of high-quality primary care.

\section{Workforce Development}

Transformation of primary care requires transformation of the primary care workforce. Addressing health disparities requires diversifying the primary care workforce to fully represent the communities served, including racial, ethnic, and linguistic minorities, individuals from low-income and working-class backgrounds, and those from rural communities. A commitment to understanding and addressing clinician burnout and its underlying causes is fundamental. Research is needed on how to effectively use teams of professionals-both clinicians and non-clinician staff-to improve access, efficiency, and coordination of care. We need evidence identifying the optimal use of community health workers or promotores, patient navigators, and other community-based professionals. We need tested models of care that integrate behavioral and mental health professionals into primary care practices either as a shared community resource or embedded in practices. Frontline clinicians need to be included as partners in research and be engaged in codesigning real world solutions.

\section{Tools and Methods for Primary Care Research}

\section{Innovation in Primary Care Research}

Achieving the universal implementation of the core attributes of primary care will require innovative approaches to primary care research. We need to understand what works for which populations and in which contexts as well as how to successfully implement new models of care. By integrating implementation science with quality improvement, we can generate practice-based evidence while implementing new models and approaches to care delivery. Agile implementation methods and rapid-cycle evaluation can increase the probability that proposed interventions will lead to measurable improvements. Methods drawn from complexity science can inform interventions that address the complex needs of individuals receiving care in the context of their lives and communities from a health care system that is also complex. Multilevel interventions align the work of health systems, practices, patients, caregivers, communities, and public health. The long timeframes for conducting research are at odds with practices' needs for timely evidence for decision making, but continuous learning and prespecified interim results of studies could help. The goal is to discover interventions that result in sustainable changes.

\section{Data for Evidence Generation}

The next generation of primary care research can be nurtured by the development of data to assess primary care nationally and locally. Data development is also needed to better manage both individuals and populations. Practice-based research networks (PBRNs), to which AHRQ provided infrastructure and capacity-building funding over many decades, can play a critical role in assuring that practice-based evidence from real world primary care data is available to guide evidence-based practice. Data on clinical processes and health outcomes can be harnessed through optimizing the use of electronic health records, patient registries, health information exchanges, and patient-generated health data. Predictive analytics have the potential to support risk stratification, and to identify and target those most likely to benefit from specific services and supports. Tools are needed that allow clinicians and researchers easy access to and use of patient-level, aggregated, and contextual data for quality improvement, population health, and health services research.

\section{CONCLUSION}

Realizing the potential of primary care will require wise investments in primary care research. There is already a large body of primary care research and tools on which to build. ${ }^{17-20}$ Newly generated evidence needs to be rapidly incorporated into the design of the delivery system, clinical care, and community interventions. Investments in evidenceinformed primary care redesign from a robust agenda of primary care research can catalyze progress to achieving the quintuple aim-improved health outcomes, increased value, better patient and clinician experience, and health equity. Primary care research can provide the evidence to help stem the twin epidemics of clinician burnout and lack of trust in the health system. Actualizing this vision will require a concerted and coordinated effort by policy makers, researchers, clinicians, and community members and a commitment to ensuring people and communities have ready access to primary care. Research can show the path forward, but all will need to walk the path.

Read or post commentaries in response to this article.

Key words: primary health care; primary care; health system performance; patient-centered care; health equity; quality of care

Submitted August 18, 2021; submitted, revised, November 14, 2021; accepted December 1, 2021

Disclaimer: The findings and conclusions in this document are those of the authors, who are responsible for its content, and do not necessarily represent the views of the Agency for Healthcare Research and Quality (AHRQ). No statement in this report should be construed as an official position of AHRQ or the US Department of Health and Human Services.

Acknowledgments: We thank Elisabeth Kato for her thoughtful feedback on the commentary.

\section{References}

1. Andrasfay T, Goldman N. Reductions in 2020 US life expectancy due to COVID-19 and the disproportionate impact on the Black and Latino populations. Proc Natl Acad Sci USA. 2021;118(5):e2014746118. 10.1073/ pnas. 2014746118

2. Mendel P, Gidengil C, Tomoaia-Cotisel A, et al. Health Services and Primary Care Research Study: Comprehensive Report. RAND Corporation; 2021.

3. National Academies of Sciences, Engineering, and Medicine. Implementing High-Quality Primary Care: Rebuilding the Foundation of Health Care. National Academies Press; 2021. https://www.nationalacademies.org/our-work/ implementing-high-quality-primary-care 
4. World Bank. Walking the Talk: Reimagining Primary Health Care After COVID-19. World Bank; 2021. https://www.worldbank.org/en/topic/health/publication/ walking-the-walk-reimagining-primary-health-care-after-covid-19-a-healthnutrition-and-population-global-practice-flagsh

5. Montori VM, Hargraves I, McNellis RJ, et al. The care and learn model: a practice and research model for improving healthcare quality and outcomes. J Gen Intern Med. 2019;34(1):154-158. 10.1007/s11606-018-4737-7

6. World Health Organization. Framework on integrated, people-centered health services; report by the Secretariat. Document A69/39. Published Apr 15, 2016. Accessed Jul 20, 2021. https://apps.who.int/gb/ebwha/pdf_files/ WHA69/A69_39-en.pdf

7. National Healthcare Quality and Disparities Report Chartbook on Person- and Family-Centered Care. AHRQ Pub. No. 16(17)-0015-9-EF. Agency for Healthcare Research and Quality; 2016. https://www.ahrq.gov/research/findings/ nhqrdr/chartbooks/personcentered/index.html

8. Dymek C, Johnson M Jr, McGinnis P, et al. Clinical-Community Relationships Measures Atlas. AHRQ Publication No. 13-0041-EF. Agency for Healthcare Research and Quality; 2013. https://www.ahrq.gov/prevention/resources/ chronic-care/clinical-community-relationships-measures- atlas/index.html

9. Coleman K, Wagner E, Schaefer J, Reid R, LeRoy L. Redefining Primary Care for the 21st Century; White Paper. AHRQ Publication No. 16(17)-0022-EF. Agency for Healthcare Research and Quality; 2016. https://www.ahrq.gov/ sites/default/files/wysiwyg/professionals/systems/primary-care/workforcefinancing/white_paper.pdf

10. Robinson EJ, Embi PJ, Raju R, Yu Y. A call for action to achieve health equity. Agency for Healthcare Research and Quality blog. August 16, 2021 Accessed Oct 12, 2021. https://www.ahrq.gov/news/blog/ahrqviews/achievehealth-equity.html

11. Agency for Healthcare Research and Quality. AHRQ's digital solutions to support care transitions challenge. Accessed Oct 12, 2021. https://www.ahrq. gov/mcctransitions-challenge/index.html
12. New Models of Primary Care Workforce and Financing Executive Summary. AHRQ Publication No. 16(17)-0046-9-EF. Agency for Healthcare Research and Quality; 2016. https://www.ahrq.gov/ncepcr/primary-care-research/ workforce-financing/index.html

13. Schottenfeld L, Petersen D, Peikes D, et al. Creating Patient-Centered TeamBased Primary Care. AHRQ Pub. No. 16-0002-EF. Agency for Healthcare Research and Quality; 2016. https://pcmh.ahrq.gov/page/creating-patientcentered-team-based-primary-care

14. DeVoe JE. Primary care is an essential ingredient to a successful population health improvement strategy. J Am Board Fam Med. 2020;33(3):468-472. 10.3122/jabfm.2020.03.190294

15. Tapp H, White L, Steuerwald M, Dulin M. Use of community-based participatory research in primary care to improve healthcare outcomes and disparities in care. J Comp Eff Res. 2013;2(4):405-419.

16. State leadership fact sheet. Primary Care Collaborative. Published Mar 2020. Accessed Oct 12, 2021. https://www.pcpcc.org/resource/state-leadership-factsheet-march-2020

17. Arora S, Thornton K, Murata G, et al. Outcomes of treatment for hepatitis C virus infection by primary care providers. N Engl J Med. 2011;364(23): 2199-2207. 10.1056/NEJMoa1009370

18. TeamSTEPPS for Office-Based Care Version. Agency for Healthcare Research and Quality; 2018. https://www.ahrq.gov/teamstepps/officebasedcare/index.html

19. The EvidenceNOW Model: Providing External Support for Primary Care. Agency for Healthcare Research and Quality; 2019. https://www.ahrq.gov/evidence now/about/evidencenow-model.html

20. About Learning Health Systems. Agency for Healthcare Research and Quality; 2019. https://www.ahrq.gov/learning-health-systems/about.html 